\title{
Novel three-dimensional cultures provide insights into thyroid cancer behavior
}

\author{
Mason A Lee1,2, Kensey N Bergdorf3, Courtney J Phifer', Caroline Y Jones¹, Sonia Y Byon'1, Leah M Sawyer, \\ Joshua A Bauer ${ }^{5}$ and Vivian L Weiss ${ }^{1}$
}

1Department of Pathology, Microbiology, and Immunology, Vanderbilt University Medical Center, Nashville, Tennessee, USA

zUniversity School of Nashville, Nashville, Tennessee, USA

3Department of Pharmacology, Vanderbilt University, Nashville, Tennessee, USA

${ }^{4}$ Department of Cell and Developmental Biology, Vanderbilt University, Nashville, Tennessee, USA

${ }^{5}$ Department of Biochemistry, Vanderbilt University, Nashville, Tennessee, USA

Correspondence should be addressed to V L Weiss: Vivian.I.weiss@vanderbilt.edu

\begin{abstract}
Thyroid cancer has the fastest growing incidence of any cancer in the United States, as measured by the number of new cases per year. Despite advances in tissue culture techniques, a robust model for thyroid cancer spheroid culture is yet to be developed. Using eight established thyroid cancer cell lines, we created an efficient and cost-effective $3 \mathrm{D}$ culture system that can enhance our understanding of in vivo treatment response. We found that all eight cell lines readily form spheroids in culture with unique morphology, size, and cytoskeletal organization. In addition, we developed a high-throughput workflow that allows for drug screening of spheroids. Using this approach, we found that spheroids from K1 and TPC1 cells demonstrate significant differences in their sensitivities to dabrafenib treatment that closely model expected patient drug response. In addition, K1 spheroids have increased sensitivity to dabrafenib when compared to monolayer K1 cultures. Utilizing traditional 2D cultures of these cell lines, we evaluated the mechanisms of this drug response, showing dramatic and acute changes in their actin cytoskeleton as well as inhibition of migratory behavior in response to dabrafenib treatment. Our study is the first to describe the development of a robust spheroid system from established cultured thyroid cancer cell lines and adaptation to a high-throughput format. We show that combining 3D culture with traditional 2D methods provides a complementary and powerful approach to uncover drug sensitivity and mechanisms of inhibition in thyroid cancer.
\end{abstract}

\section{Introduction}

The incidence of thyroid cancer is increasing at a faster rate than any other cancer in American women. Sixty percent of new cases are in women younger than 55 years of age (Brose et al. 2014). Approximately 100 million people in the United States have thyroid nodules, though only up to $15 \%$ are estimated to be cancerous (Gharib
2004). Most thyroid cancers can be successfully treated with resection and radioactive iodine, but tumors recur in $20 \%$ of patients and metastasize in approximately $10 \%$ (Grebe \& Hay 1996, Schlumberger 1998). For patients with distant metastases, $60 \%$ become iodide refractory during the course of their disease, leaving them with
(C) 2020 Society for Endocrinology Published by Bioscientifica Ltd. Printed in Great Britain
Endocrine-Related Cancer (2020) 27, 111-121 
limited treatment options (Anderson et al. 2013, Brose et al. 2014). In addition, aggressive anaplastic thyroid carcinomas (ATCs) can develop from well-differentiated thyroid cancers and represent a deadly disease with an $\sim 4$-month overall average survival. Our current culture methods do not account for many in vivo properties of tumors, making them relatively inefficient for developing therapeutics. It is critical to develop novel thyroid cancer culture methods that more closely mimic patient responses in order to identify therapies that can be used in treatment-resistant disease.

The recently FDA-approved BRAF/MEK inhibitor combination therapy represents a major advancement in care for ATC patients (Subbiah et al. 2018). The BRAF oncogene encodes a component of the MAPK/ ERK signaling pathway, which controls growth and cell division (McCain 2013). Activating BRAF mutations are common in thyroid cancer, which makes it an attractive drug target. Unfortunately, only $30-40 \%$ of BRAF-mutant thyroid cancers respond to the BRAF inhibitor dabrafenib (Cabanillas et al. 2015). However, combination BRAF/MEK inhibition leads to increased treatment response in ATC patients (Subbiah et al. 2018), likely due to the blockade of paradoxical ERK activation (Lito et al. 2013). This treatment is only available for patients with BRAFV600E-mutant tumors, as it may cause disease progression in tumors with WT BRAF. Indeed, there are few other effective treatments for aggressive thyroid cancers, despite our knowledge of the important signaling pathways (MAPK, Wnt, PI3K, and others) that are critical for tumor development and progression. A near-in vivo culture method that would allow for rapid and facile drug testing across tumors with a heterogeneous mutational background would revolutionize thyroid cancer drug discovery. Significantly, treatment of patient-derived organoids has been shown to closely correspond with patient response to therapy (Vlachogiannis et al. 2018). Development of a cost-effective method for thyroid cancer organoid/spheroid culture that closely recapitulates patient responses may lead to novel therapeutic discoveries for BRAF-wildtype disease.

In the current study, we show that eight thyroid cancer cell lines readily form spheroids that represent unique morphology and drug sensitivity. In addition, we find significant differences in the degree of drug sensitivity between the $2 \mathrm{D}$ and $3 \mathrm{D}$ systems. Our results suggest that 2D culture of cell lines provides a useful system for uncovering mechanisms of behavior, whereas the $3 \mathrm{D}$ system provides a more accurate platform for translational drug screening.

\section{Materials and methods}

\section{Thyroid cancer cell lines}

Cell lines were purchased from commercial vendors (K1, Sigma Aldrich; MDA-T32 and MDA-T68 lines, American Type Culture Collection). The TPC- 1 line was obtained from Dr Adel El-Naggar (University of Texas MD Anderson Cancer Center, Houston, TX, USA). THJ-11T, THJ-16T, THJ-21T, and THJ-29T were obtained from their creator, Dr John Copland (Mayo Clinic, Jacksonville, FL, USA).

Thyroid cancer cells were authenticated using STRS analysis and were maintained and used experimentally at $<20$ passages. Cell lines were grown in media containing $10 \%$ fetal bovine serum (ThermoFisher Scientific) and RPMI (VWR). Media was also supplemented with 1\% penicillin-streptomycin (Sigma), 1× MEM Non-Essential Amino Acid (VWR), and $1 \mathrm{mM}$ sodium pyruvate (Vanderbilt Molecular Biology Resource).

\section{Spheroid disc culture}

Cells were mixed with 75\% Matrigel in completed media and 100-300 $\mu \mathrm{L}$ drops (discs) were added to each well of a pre-warmed 6-well plate. Plates were incubated for $10 \mathrm{~min}$ to allow the Matrigel to solidify. Discs were then covered with warm complete media containing 10\% FBS plus B27 supplement (Gibco 17504044). Media was changed every 2-3 days.

\section{Semi-solid spheroid culture}

Cells were mixed with 5\% Matrigel in complete media with $12 \%$ FBS. Cells were plated at 8000 cells/well in 500 $\mu \mathrm{L}$ media/Matrigel/FBS + drug in each well of a 24-well ultra-low attachment plate (Corning 3473). Fresh, complete media with $12 \% \mathrm{FBS}+5 \%$ Matrigel was added drop-wise to the culture once per week.

\section{Brightfield and phase-contrast microscopy}

Brightfield images of spheroids were acquired on a Leica DMi1 inverted microscope with a $4 \times$ objective or an EVOS FL inverted microscope with a $20 \times$ objective. Phasecontrast images were acquired on a Zeiss Axiophot using an Olympus DP72 camera attachment. The objective was a $20 \times$ plan neofluor objective (dry) with a phase 2 condenser. 


\section{H\&E staining}

After 8 days in culture, spheroid discs were fixed with $10 \%$ formaldehyde $+1 \%$ glutaraldehyde (to maintain Matrigel integrity). Discs were then processed and dehydrated in a standard tissue processor and paraffin embedded. Tissue sections $(4 \mu \mathrm{m})$ were stained with hematoxylin and eosin using standard protocols.

\section{Monolayer immunofluorescence}

Thyroid cancer cells were seeded onto 24-well tissue culture plates $(50,000$ cells per well) containing coverslips and allowed to attach for $24 \mathrm{~h}$. Cell were then fixed for 20 min with 4\% formaldehyde in PBS. For staining the actin cytoskeleton with phalloidin, 4\% formaldehyde in cytoskeleton buffer (10 mM MES pH 6.1, $138 \mathrm{mM} \mathrm{KCl,}$ and 2 mM EGTA plus sucrose $(0.114 \mathrm{~g} / \mathrm{mL}))$ was used. Cells were then washed with $0.1 \%$ TBST wash buffer (TBS (20 $\mathrm{mM}$ Tris $\mathrm{Cl} \mathrm{pH} 7.4$ and $150 \mathrm{mM} \mathrm{NaCl}$ ) containing $0.1 \%$ Triton X-100) at RT, permeabilized with 0.5\% TBST (TBS containing $0.5 \%$ Triton X-100) for $10 \mathrm{~min}$ at RT, washed with $0.1 \%$ TBST, and incubated for $1 \mathrm{~h}$ at RT in blocking buffer (0.1\% TBST plus 2\% BSA). Cells were then incubated with mouse anti-tubulin MAB (1:1000; Vanderbilt Protein and Antibody Resource; $1 \mathrm{mg} / \mathrm{mL}$ stock) or mouse anti$\beta$-catenin MAB (1:500; Vanderbilt Protein and Antibody Resource; $1 \mathrm{mg} / \mathrm{mL}$ stock) in blocking buffer overnight at $4^{\circ} \mathrm{C}$. After incubation, cells were washed three times for 20 min per wash with $0.1 \%$ TBST and incubated with Alexa Fluor 488- or Alexa Fluor 546-conjugated secondary antibodies (Abcam; 1:300) diluted in blocking buffer for $1 \mathrm{~h}$ at RT. For actin staining, Alexa Fluor 568 Phalloidin (1:1000; ThermoFisher Scientific) in PBS was used. Cells were then washed with PBS, incubated with Hoechst in TBS (1:1000; ThermoFisher Scientific) for 5 min, and rinsed with PBS before mounting in ProLong Gold Antifade Reagent (Invitrogen). Images were acquired using a CoolSNAP ES camera mounted on a Nikon Eclipse 80 i fluorescence microscope with $40 \times$ objective.

\section{Spheroid immunofluorescence}

Spheroids were fixed in 4\% formaldehyde in PBS for 30 min at $4^{\circ} \mathrm{C}$ and then transferred into $2.0 \mathrm{~mL}$ tubes, rinsed three times in $0.1 \%$ TBST, permeabilized with $0.5 \%$ TBST for $30 \mathrm{~min}$ on a rotator at RT, rinsed with $0.1 \%$ TBST, and incubated in blocking buffer for $1 \mathrm{~h}$ on a rotator at RT. Spheroids were then incubated with mouse anti- $\beta$ catenin antibody (1:500, Vanderbilt Protein and Antibody Resource) or rabbit anti-E-cadherin antibody (1:200, Cell
Signaling Technology) in blocking buffer overnight on a rotator at $4^{\circ} \mathrm{C}$. After incubation, spheroids were washed three times for 30 min per wash in $0.1 \%$ TBST and incubated for 2-3 h on a rotator at RT with Cy3-conjugated anti-mouse secondary antibody (1:500, Jackson Immune) or Cy3-conjugated anti-rabbit secondary antibody (1:500, Abcam) diluted in blocking buffer. Spheroids were rinsed in $0.1 \%$ TBST and then incubated with Alexa Fluor 568 Phalloidin (1:1000; ThermoFisher Scientific) and Hoescht (1:1000) in $0.1 \%$ TBST for $20 \mathrm{~min}$ on a rotator at RT. Spheroids were rinsed $1 \times$ in $0.1 \%$ TBST. Spheroids were put into $1 \times$ PBS (Vanderbilt Molecular Biology Resource) and then mounted on microscope slides with ProLong Gold Antifade Reagent (Invitrogen). Images were acquired using a Nikon Spinning Disk microscope with Andor DU-897 EMCCD camera with $561 \mathrm{~nm}$ and $405 \mathrm{~nm}$ lasers. Images were processed using ImageJ.

\section{In vitro scratch assay}

The scratch assay for K1 and TPC1 cells in the absence or presence of dabrafenib was performed as previously described (Liang et al. 2007). K1 or TPC1 cells were grown on coated 10-mm plates (ThermoFisher Scientific) until cells reached $100 \%$ confluency. A scratch was then created using a $1000 \mu \mathrm{L}$ pipette tip across the width of the plate. Images of the scratch were acquired with a Leica DMi1 MC170 inverted microscope with a $4 \times$ objective and processed on Leica Application Suite, version 4.10.0. The number of cells that migrated into the scratched area were counted. All experiments were performed in triplicate.

\section{High-throughput analysis of $\mathrm{K} 1$ and TPC1 cells/spheroids}

Utilizing the high-throughput equipment in the Vanderbilt high-throughput screening (HTS) core facility, $\mathrm{K} 1$ and TPC1 cells were resuspended at a concentration of 9500 cells/mL in complete RPMI (2D culture) or complete RPMI supplemented with 2\% Matrigel (3D culture). $30 \mu \mathrm{L}$ of cell suspension was plated per well in black 384-well cell culture microplates in the absence or presence of a cellrepellant surface (Greiner Bio-One). Cells were allowed to attach for $24 \mathrm{~h}$ prior to treatment with dabrafenib or DMSO (vehicle) in RPMI. Following $72 \mathrm{~h}$ of treatment, wells were imaged using an ImageXpress Micro XL automated high-content microscope (Molecular Devices). To assess viability, CellTiter-Glo 3D (Promega) was added to wells and mixed with the Bravo liquid handler (Velocity 11/Agilent). Per the CellTiter-Glo protocol, plates were 
placed on a shaker for 25 min before luminescence was quantified using a Synergy NEO (BioTek multi-mode plate reader).

\section{Statistical analysis}

Statistical analyses were performed using Fisher's exact test. A value of $P<0.05$ is considered statistically significant. Data for 384-well dabrafenib viability studies were analyzed using Prism 8 (GraphPad Software, Inc). To determine significance, a two-way ANOVA was performed and followed with a Sidak multiple comparisons test.

\section{Results}

\section{Staining for microtubules, actin, and $\beta$-catenin shows distinct patterns in the different thyroid cancer cell lines}

For our studies, we chose eight thyroid cancer lines (papillary thyroid cancer (PTC) cell lines: K1, MDA-T32, MDA-T68, and TPC1; and ATC cell lines: THJ-11T, THJ-16T, THJ-21T, and THJ-29T) with known driver mutations (Table 1). Brightfield images show that these lines exhibit varying morphology (Fig. 1). For example, the $\mathrm{K} 1$ and THJ-16T cells grow in a flat, epitheliod pattern, while the MDA-T68 cells have an elongated, spindle-like morphology. The thyroid cell lines also vary in both overall cell size and nuclear:cytoplasmic ratios. Microtubule immunostaining revealed differences in their distribution among cell lines with K1, TPC1, and THJ-16T having prominent Golgi-derived microtubules that fan out into the cytoplasm. In contrast, MDA-T32 and MDA-T68 cell lines show prominent microtubule bundles that traverse the length of the cells (Fig. 1). Actin staining revealed two distinctive morphologies: cells with prominent actin stress fibers (TPC1, MDA-T32, MDA-T68, THJ-11T, and $\mathrm{THJ}-21 \mathrm{~T}$ ) and cells with enriched lamellipodial

Table 1 Thyroid cancer cell lines used in this study and associated driver mutations.

\begin{tabular}{lll}
\hline \multicolumn{1}{c}{ Cell line } & & Driver mutations \\
\cline { 1 - 1 } K1 & & BRAFV600E, PI3K \\
TPC-1 & & RET/PTC fusion \\
MDA-T32 & & BRAFV60E, TERT promoter \\
MDA-T68 & & RAS \\
THJ-29T & & TERT promoter, TP53 \\
THJ-21T & & BRAFV00E, TERT promoter, TP53 \\
THJ-11T & & KRAS, TERT promoter, TP53 \\
THJ-16T & PI3K, TERT promoter, TP53, MKRN1-BRAF fusion \\
\hline
\end{tabular}

(c) 2020 Society for Endocrinology Published by Bioscientifica Ltd. Printed in Great Britain actin (K1, THJ-16T, and THJ-29T). In addition, THJ-29T cells show actin-enriched ring structures reminiscent of podosomes, which are implicated in cell migration and invasion (Linder \& Kopp 2005). Thus, the differences in cytoskeleton organization between thyroid cancer cell lines may be indicative of varying capacity to migrate, form 3D structures, and invade.

We then immunostained for $\beta$-catenin, which is part of the E-cadherin complex and is also a critical mediator of the oncogenic Wnt pathway in the nucleus (Saito-Diaz et al. 2013). We found that all of the thyroid cancer cell lines contain cytoplasmic $\beta$-catenin. The K1 and MDA-T32 lines, however, show significant staining for nuclear $\beta$-catenin, suggesting ongoing Wnt signaling in those cell lines.

\section{Thyroid cancer cell lines uniformly form spheroids in culture}

Spheroids and organoids are 3D, self-organizing multicellular structures that mimic the complex organization and function of tissues and organs (Takebe \& Wells 2019). These culture methods provide a promising new in vitro method to study cancer progression and drug sensitivity, allowing for personalized treatment approaches ( $\mathrm{Xu}$ et al. 2018). With the overall goal of a $3 \mathrm{D}$ cell culture system, we sought to grow spheroids from the eight established thyroid cancer cell lines. As these cell lines each have unique genetic features, we aimed to generate a diverse cohort of thyroid cancer spheroids to serve as the first step in drug development and screening. We found that all of the cell lines readily formed spheroids in culture (Fig. 2). Spheroid morphology and size differed dramatically between the different thyroid cancer cell lines. Brightfield imaging and H\&E staining showed that some spheroids form robust macro-follicular structures, including K1, THJ-11T, and THJ-16T. Other cell lines, such as MDA-T32 and MDA-T68, grow in a microfollicular arrangements that more closely mimic a follicular variant histologic pattern. TPC1, THJ-21T, and THJ-29T formed spheroids but demonstrated a more invasive and loosely cohesive architecture. E-cadherin staining in the spheroids also showed varying intensity (Fig. 2). Loss of E-cadherin is a hallmark of epithelial-to-mesenchymal transition, which is postulated to be critical for cancer progression (Lamouille et al. 2014). Increased cytoplasmic $\beta$-catenin has been associated with decreased E-cadherin expression and may represent increased Wnt signaling, a feature of more aggressive thyroid tumors (SastrePerona et al. 2016, Ely et al. 2018). Alternatively, loss of 

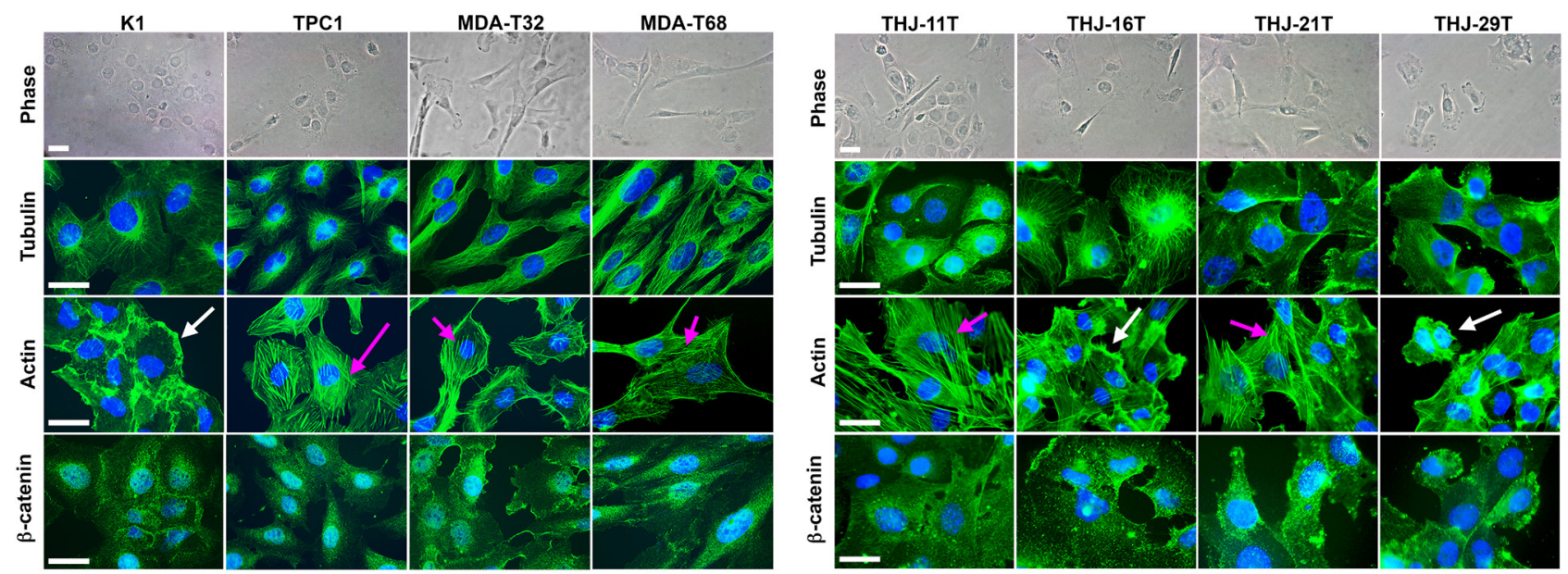

Figure 1

Characterization of eight genetically distinct thyroid cancer cell lines shows varying morphology and staining patterns for microtubules, actin, and $\beta$-catenin. Thyroid cancer cells were grown on glass coverslips, fixed, and stained with phalloidin (for filamentous actin), anti-tubulin antibody, or anti- $\beta$-catenin antibody. Cell lines in the left panel are derived from papillary thyroid carcinoma. Cell lines in the right panel are derived from anaplastic thyroid carcinoma. Top panels show phase contrast. Tubulin staining (green) shows distinct Golgi microtubules in some of the thyroid cancer lines, whereas other lines show long microtubule cables running along the length of the cells. Actin staining (green) reveals cell lines with either lamellipodial (white arrows) or stress fiber predominant patterns (pink arrows). Nuclear $\beta$-catenin (green) is observed in many cell lines, suggesting active Wnt signaling. DNA is shown by DAPI (blue). Scale bar is 20 microns.

E-cadherin could release associated $\beta$-catenin, thereby increasing its cytoplasmic levels. $\beta$-Catenin staining in all eight spheroids confirmed that E-cadherin staining is inversely correlated with the level of $\beta$-catenin (Fig. 2). The K1, THJ-11T, and THJ-16T cells exhibited prominent E-cadherin staining but showed low levels of $\beta$-catenin. In contrast, TPC1, THJ-21T, and THJ-29T spheroids showed elevated levels of $\beta$-catenin and lower levels of E-cadherin staining throughout the spheroids, consistent with their more infiltrative morphology. Of note, the cell lines with high $\beta$-catenin levels in 2D culture showed lower levels of $\beta$-catenin when grown in a spheroid format, potentially reflecting differences between a $2 \mathrm{D}$ and $3 \mathrm{D}$ tumor microenvironment.
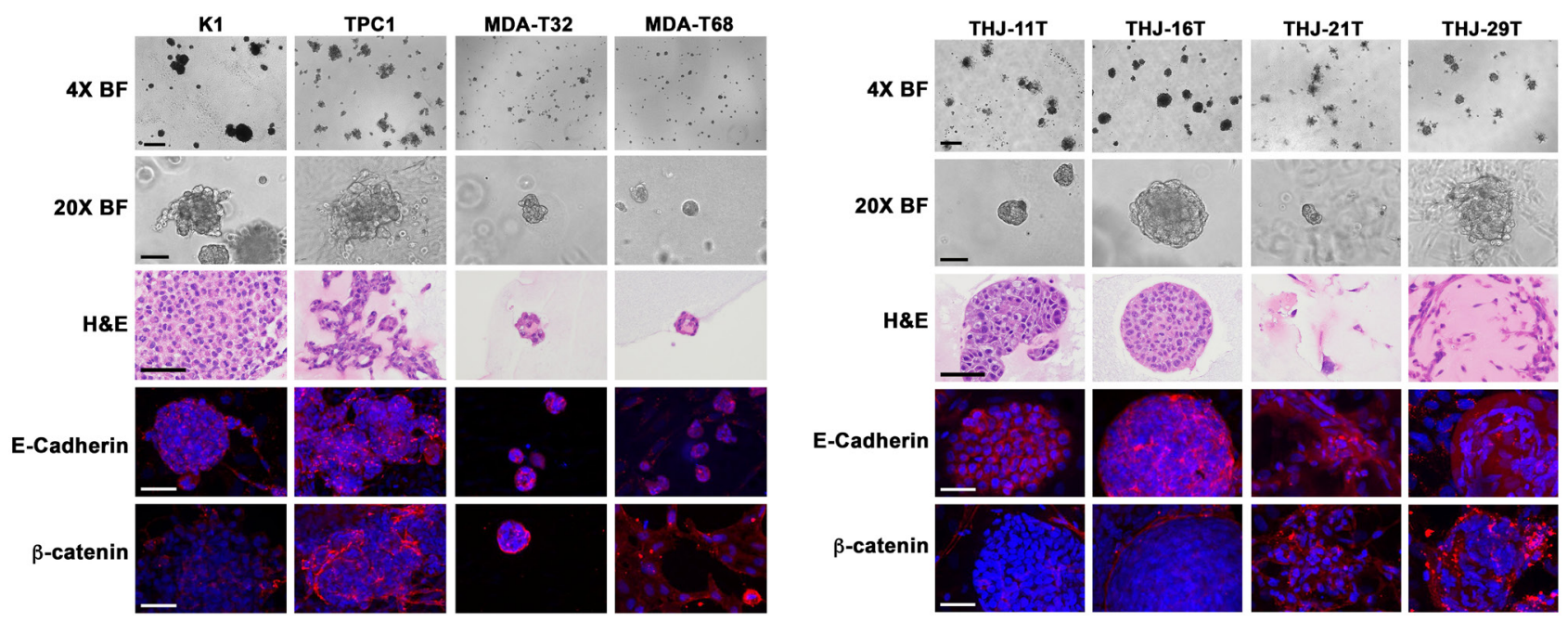

\section{Figure 2}

Thyroid cancer cell lines readily form spheroids that differ in morphology, $\beta$-catenin, and E-cadherin expression. Thyroid cancer cells were grown in Matrigel discs, fixed, and stained with anti-E-cadherin antibody or anti- $\beta$-catenin antibody. Cell lines in the left panel are derived from papillary thyroid carcinoma. Cell lines in the right panel are derived from anaplastic thyroid carcinoma. Top panels show low and high magnification brightfield (BF) images. Hematoxylin and eosin (H\&E) staining of organoid cultures highlights differences in spheroid morphology. For most thyroid cancer spheroid lines, E-cadherin staining intensity inversely correlated with the intensity of $\beta$-catenin staining. E-cadherin and $\beta$-catenin are red. DNA is blue. Scale bar is $100 \mu \mathrm{m}$ except for $4 \times \mathrm{BF}(400 \mu \mathrm{m})$. 


\section{Response of thyroid cancer spheroids to dabrafenib treatment mimics patient response}

The recent development of organoid and spheroid culture provides a near in vivo system that more accurately captures the behavior of a tumor in response to treatment (Vlachogiannis et al. 2018, Saito et al. 2019). We therefore assessed whether thyroid cancer spheroids respond to treatment with the BRAF inhibitor dabrafenib in a manner similar to patient responses. We first examined the effect of dabrafenib on the morphology of the BRAFV600E-mutant and BRAF-wild-type thyroid cancer spheroids. We found that $48 \mathrm{~h}$ dabrafenib treatment had the most dramatic effect on spheroids from the papillary thyroid carcinoma (PTC) K1 cell line, which harbors an activating BRAFV600E mutation and a PI3K mutation (Fig. 3). K1 spheroids were much smaller when grown in the presence of dabrafenib. Of note, the more aggressive BRAFV600E-mutant PTC MDA-T32 cell line, which also carries a TERT promoter mutation, showed limited response to dabrafenib. Similarly, the aggressive BRAFV600E-mutant ATC cell line (THJ-21T), which harbors TERT promoter and TP53 mutations, also showed limited response to dabrafenib. The remaining cell lines, which were BRAF-wildtype, did not show response to dabrafenib. It is not surprising that the more aggressive BRAFV600E-mutant thyroid cancers, with TERT promoter mutations, were less susceptible to dabrafenib therapy alone (Xing et al. 2014, Liu \& Xing 2016). Clinical trials have shown limited response of aggressive BRAFV600E-mutant thyroid cancers to single agent dabrafenib therapy. Prolonged treatment of K1 and TPC1 spheroids with dabrafenib furthered the robust drug effects. As shown in Fig. 3B and C, K1 spheroids exposed to dabrafenib for 8 days were dramatically smaller than vehicle-treated controls. In contrast, BRAF-wildtype TPC1 spheroids showed a significant increase in spheroid size. This finding is consistent with clinical findings, as BRAFwildtype tumors often show rapid progression when treated with BRAF inhibitors. While the mechanism of this robust cancer progression following dabrafenib therapy is unknown, we observed a highly significant and robust increase in the mitotic index for TPC1 cells grown in dabrafenib for $48 \mathrm{~h}$ (Supplementary Fig. 1, see section on supplementary materials given at the end of this article). Evaluation of the mitotic index for K1 cells when grown for $48 \mathrm{~h}$ with dabrafenib showed only a slight increase.

\section{Establishment of a high-throughput thyroid cancer spheroid assay for drug screening}

The advantages of screening for drug sensitivity using 2D cultures include the ability to rapidly test a large number of conditions and concentrations, the ability to use cells with defined genetic lesions, and the availability of a large number of cell lines for testing. However, cancer cells grown on a flat surface respond very differently to drugs when compared to the original tumor from which they are derived. As such, we sought to adapt 3D thyroid cancer spheroid culture methods to an automated high-throughput 384-well format. High-throughput screening provides the opportunity for rapid, replicable collection of data for many drugs. The use of the Agilent Bravo automated pipette allows drugs to be added to 384 wells at once or to be stamped in for creation of replicates. To further the ability of this system, all work was done in clear-bottom plates. This enabled imaging with the ImageXpress Micro XL, an automated high-content imager, which aided in
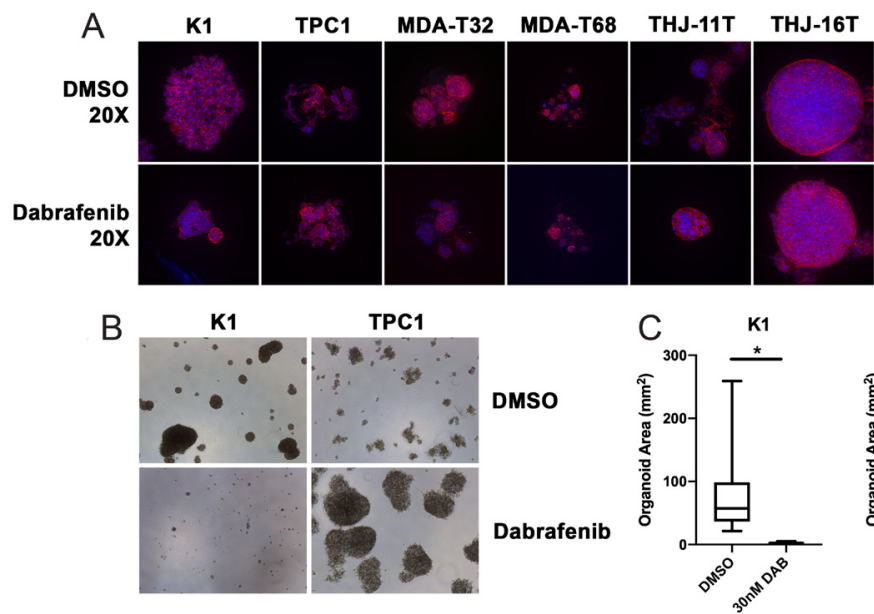

\section{Figure 3}

Effect of dabrafenib, a BRAF inhibitor, on spheroid size. (A) Thyroid cancer cell spheroids were treated with $30 \mathrm{nM}$ dabrafenib for $48 \mathrm{~h}$, stained for actin (phalloidin), and imaged using a spinning disk confocal microscope. 20x images do not show a dramatic change in morphology or size with the exception of $\mathrm{K} 1$, which show a significant decrease in size. (B) Brightfield images of $\mathrm{K} 1$ and TPC1 spheroids grown with vehicle or $30 \mathrm{nM}$ dabrafenib for eight days reveal long-term effects on spheroid size. (C) Analysis of organoid size (area in $\mathrm{mm}^{2}$ ) following treatment with vehicle (DMSO) or $30 \mathrm{nM}$ dabrafenib for 8 days demonstrates significantly decreased K1 organoid size and significantly increased TPC1 organoid size with dabrafenib $\left({ }^{*} P<0.0001\right)$. https://erc.bioscientifica.com https://doi.org/10.1530/ERC-19-0374
(C) 2020 Society for Endocrinology Published by Bioscientifica Ltd. Printed in Great Britain 
confirmation of findings from our viability assay. We were also able to observe any phenotypic changes in the spheroids following drug exposure. We found that both K1 and TPC1 spheroids could be adapted to the HTS format with minimal modifications, and we could obtain growth inhibition curves for their sensitivity to dabrafenib (Fig. 4A and B). Growth inhibition was also confirmed with high-content imaging (Fig. 4C). K1 spheroids were more sensitive to dabrafenib than monolayer $\mathrm{K} 1$ cells $(\mathrm{EC} 50=0.8 \mathrm{nM}$ and $1.8 \mathrm{nM}$, respectively) (Fig. 4A). Neither TPC1 spheroids nor monolayer cells were inhibited by dabrafenib (Fig. 4B).

\section{A K1 Growth Inhibition Curve}

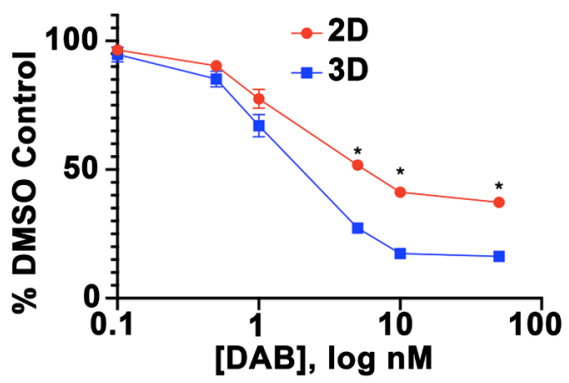

\section{B TPC1 Growth Inhibition Curve}

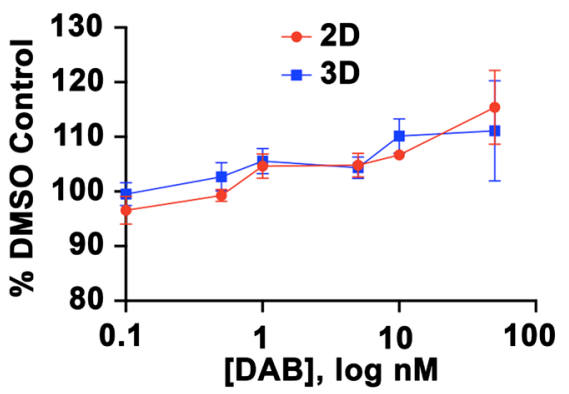

C

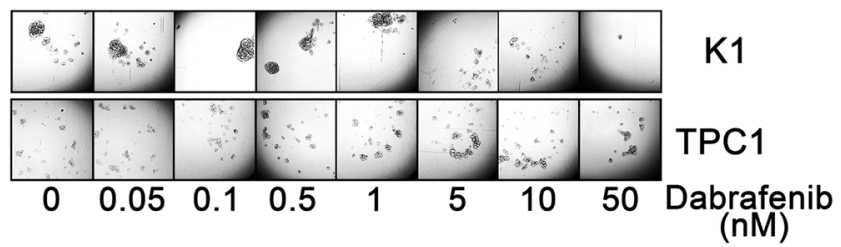

Figure 4

Thyroid cancer spheroid culture can be readily adapted for high throughput drug screening. K1 and TPC1 cells were seeded onto a 384-well plate for monolayer or spheroid culture and exposed to varying concentrations of dabrafenib. Viability was assessed after 72 h. (A) K1 spheroids were significantly more sensitive to dabrafenib treatment than monolayer $\mathrm{K} 1$ cells ( $* P<0.0001)$. (B) In contrast, dabrafenib showed no significant effect on the growth of TPC1 cells in both monolayer culture and as spheroids $(P=0.8749)$. (C) High-content imaging confirms a decrease in size and viability of K1 spheroids, as well as consistent presence of TPC1 spheroids.
Dabrafenib treatment shows differential effects on the actin cytoskeleton of the BRAF and RAS mutant cells lines, K1 and TPC1

Given our findings that there are differences in the morphology and actin cytoskeleton of various cell lines and the variable response of thyroid cancers to dabrafenib treatment, we sought to determine whether dabrafenib sensitivity could be associated with changes in the actin cytoskeleton. Using fluorescinated phalloidin for staining actin filaments, we found that treatment of $\mathrm{K} 1$ cells with dabrafenib over a period of $48 \mathrm{~h}$ induces a shift from a primarily lamellipodial actin phenotype to a stress fiber phenotype with no observable peripheral actin staining (Fig. 5A and B). Significantly, these changes can be readily observed within an hour of dabrafenib treatment. In untreated TPC1 cells, the majority contain prominent stress fibers with observable peripheral actin staining (mixed phenotype; Fig. 5C and D). TPC1 cells treated with dabrafenib over $48 \mathrm{~h}$ still primarily exhibited a mixed phenotype, but there was a significant decrease in the percent of cells containing predominantly stress fibers and a subsequent increase in cells with the actin lamellipodial pattern. No significant changes in actin were identified with vehicle (DMSO) treatment. These results suggest that the effect of dabrafenib on the actin cytoskeleton occurs rapidly, prior to alterations in gene transcription, and is therefore likely via a direct mechanism. Furthermore, the opposite effects of dabrafenib on the actin cytoskeleton of K1 cells (BRAFV600E mutant) and TPC1 cells (RET-PTC fusion) are consistent with the opposite effect of dabrafenib on thyroid cancers with mutations in BRAFV600E vs other driver alterations.

Although dabrafenib had a noticeably dramatic effect on the actin cytoskeleton of K1 and TPC1 cells grown in monolayer culture, the effect in the spheroids was much more subtle (Fig. 5E and Supplementary Videos 1-4). Taken together, these data indicate that the effects of dabrafenib on cell-cell interaction and organization were more readily assessed in the thyroid spheroid cultures, while the effects of dabrafenib on the individual cells within the spheroids were less obvious than those in the $2 \mathrm{D}$ system.

\section{Dabrafenib-induced changes in the actin cytoskeleton of K1 and TPC1 cells parallel changes in their migration rates}

Because lamellipodial actin is associated with enhanced cell motility (Rottner et al. 2017), we examined whether 

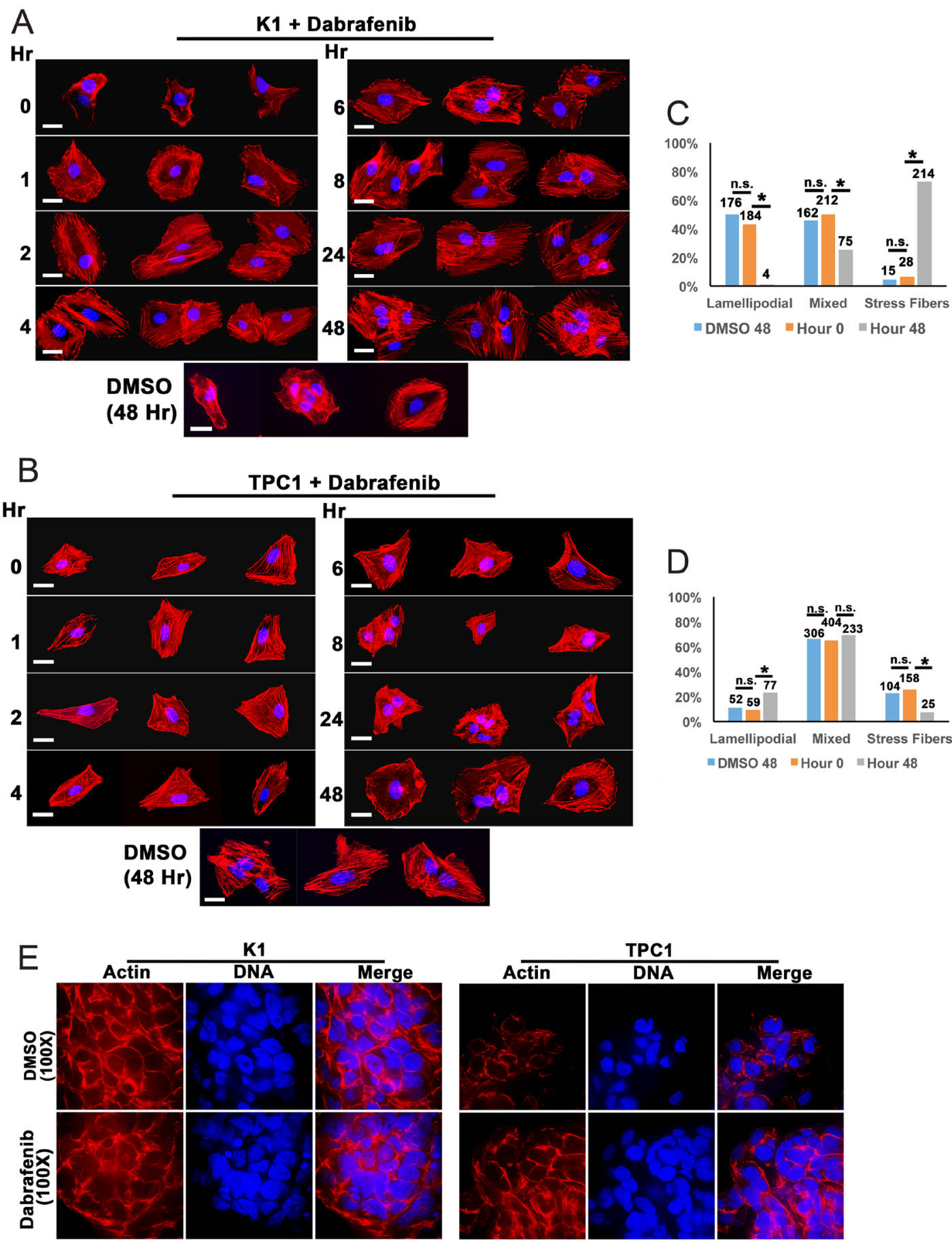

Figure 5

BRAF inhibition induces changes in actin cytoskeleton of two thyroid cancer lines. (A) K1 (BRAFV600E mutant) cells were treated with $30 \mathrm{nM}$ dabrafenib or DMSO over $48 \mathrm{~h}$, fixed at the indicated time, and stained for actin (red) and DNA (DAPl; blue). Cells show a predominantly lamellipodial phenotype, with actin enriched in the leading edge of the cell in the absence of dabrafenib. Following dabrafenib treatment, the actin cytoskeleton is converted to a primarily stress fiber phenotype that exhibits little to no observable peripheral actin. (B) TPC1 (RET-PTC fusion) cells in the absence of dabrafenib show a predominantly mixed phenotype with both stress fibers and peripheral actin. The percentage of cells with predominantly lamellipodial actin increases over $48 \mathrm{~h}$ with dabrafenib treatment. No significant change in actin is identified with DMSO treatment. Each panel contains three representative cell images from three independent replicates. Scale bar is $20 \mu \mathrm{m}$. (C and D) Quantification of the number of K1 and TPC1 cells with predominantly lamellipodial, mixed, or predominantly stress fiber phenotypes prior to and after $48 \mathrm{~h}$ dabrafenib or DMSO treatment. The number of cells scored is indicated for each phenotype and condition. n.s. non-significant, $* P<0.0001$. (E and Supplementary Videos $1-4$ ) Confocal imaging reveals a subtle effect of dabrafenib on the actin cytoskeleton of K1 and TPC1 cells, even at 100× magnification. 
the dabrafenib-induced changes observed in the actin cytoskeleton of $\mathrm{K} 1$ and TPC1 cells affected their ability to migrate. An in vitro scratch assay is a well-established, straightforward method to measure cell migration on a flat surface (Liang et al. 2007). We found that treatment of $\mathrm{K} 1$ cells with dabrafenib significantly reduced their migratory rates (Fig. 6A and B). In contrast, dabrafenib promoted slightly increased migration of TPC1 cells into the scratched area. These results are consistent with our actin staining data, as dabrafenib induces a less migratory stress fiber phenotype in $\mathrm{K} 1$ cells and a more motile lamellipodial phenotype for TPC1 cells.

\section{Discussion and conclusions}

This study represents a detailed molecular characterization of eight thyroid cancer cell lines with distinct genetic profiles and derived spheroids. We show that the thyroid cancer lines differ dramatically in their morphologies and size as evidenced by differences in microtubule and actin staining patterns. In addition, many of the cell lines show nuclear $\beta$-catenin staining, suggesting some level of oncogenic Wnt signaling that may play an important role in pathogenesis (SastrePerona et al. 2016, Nusse \& Clevers 2017). Differences in actin staining may suggest differences in migratory behavior or invasiveness. Intriguingly, the K1 (BRAFV600E_ mutant) cell line undergoes rapid and dramatic changes in actin staining when treated with dabrafenib, shifting to the less invasive stress fiber actin phenotype. The RETPTC fusion cell line TPC1 exhibited the opposite effect when treated with dabrafenib, shifting to a more invasive lamellipodial actin phenotype. These actin changes were correlated with long-term changes in spheroid growth, as well as functional changes in cellular capacity to migrate in the scratch assay. Overall, these actin changes may represent a major mechanism by which dabrafenib mediates its effects in vivo.

Organoids and spheroids have been shown to be better models for studying drug sensitivity than cells grown as a monolayer (Clevers 2016). Here, we demonstrate that we are able to grow spheroids from all eight thyroid cancer cell lines tested. These spheroids have distinct morphologies, sizes, and staining patterns for E-cadherin and $\beta$-catenin, likely reflecting differences in their original histologic subtype and tumorigenic potential. Our work shows that two spheroid cultures derived from K1 and TPC1 cell lines accurately recapitulate the in vivo response of tumors with similar genetic composition. The BRAF-mutant
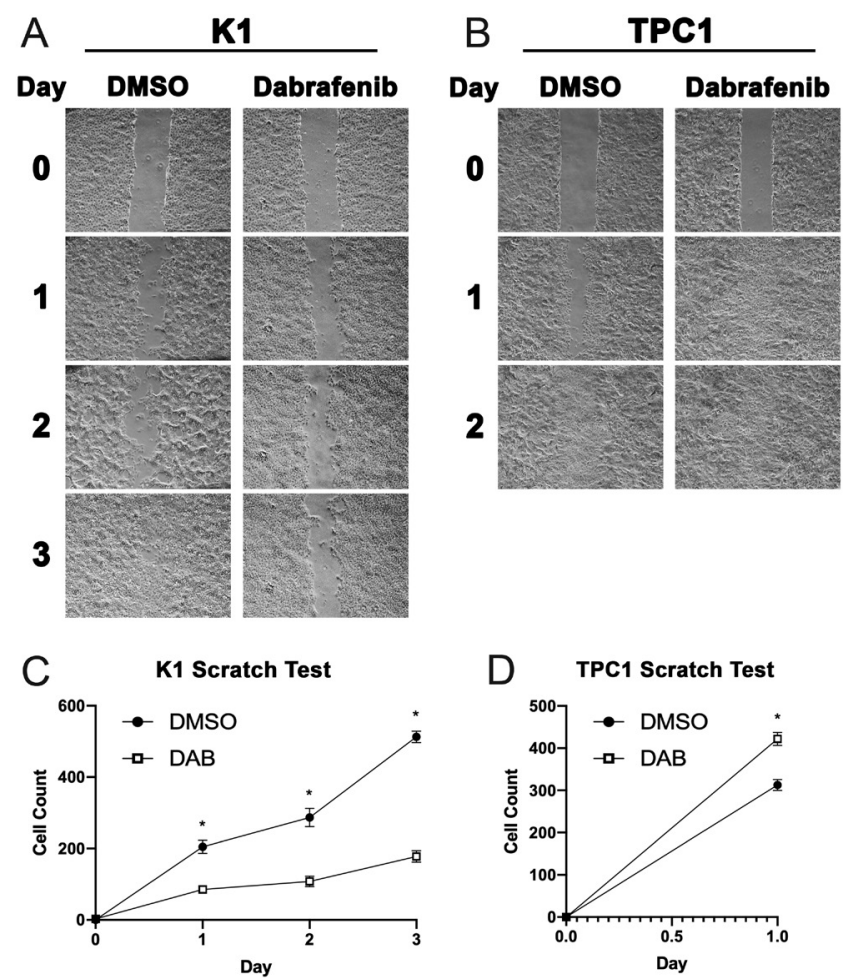

\section{Figure 6}

Changes in the capacity of K1 and TPC1 cells to migrate in a scratch assay upon BRAF inhibitor treatment parallel changes in their actin cytoskeleton. Scratches were made across the bottom of dishes of confluent (A) K1 and (B) TPC1 cells were treated with vehicle (DMSO) or 30 $\mathrm{nM}$ dabrafenib, and brightfield images of the scratch were obtained at the indicated times. (C and D) Quantification of cell migration was performed by counting the number of cells migrated into the gap at each timepoint $\left({ }^{*} P<0.05\right)$.

K1 spheroids were significantly more sensitive to dabrafenib treatment than monolayer counterparts, while the RET-PTC fusion TPC1 spheroids demonstrated increased growth following dabrafenib treatment. In the clinic, dabrafenib is contraindicated for individuals with BRAF-wildtype thyroid cancer, as it may promote tumor growth. In addition, we found that dabrafenib increases the number of mitotic TPC1 cells in the 2D system. Thus, it is possible that dabrafenib stimulates TPC1 cell division in addition to increasing their migratory behavior.

In order to facilitate efficient drug screening for thyroid cancer, we report the first adaptation of a thyroid cancer spheroid system to a high throughput 384-well format. There are numerous advantages to studying thyroid cancer in this 3D, 384-well format. First, as 3D culture better recapitulates physiologic disease, drug screening results in 3D may provide more accurate response information than $2 \mathrm{D}$ to predict in vivo testing. Therefore, less false positives would be expected in 3D drug screens than in 2D drug screens and may accelerate discoveries. 
Second, miniaturizing the 3D spheroids to 384-well plates allows for a wider range of drugs/compounds to be tested, increasing the number of gene targets. Using high-throughput assays and automation also provides the ability to screen many cell lines/spheroids to generate 'drug profiles' that can be correlated with genetic information and/or biomarkers of disease. Third, drug/compound library screening in 3D models could lead to acceleration in validating drug response/resistance mechanisms in these models that may allow for the identification of drug repurposing, drug combinations (e.g. dabrafenib+Wnt or PI3K inhibitor), or novel drug targets for the disease. Finally, 3D models in multi-well plates can allow for further studies of biology using functional genomics screening (CRISPRi or CRISPRa), for co-culturing tumor and stromal cells to study microenvironments, or co-culturing spheroids and activated immune cells to test immune modulating agents.

$3 \mathrm{D}$ culture is an attractive, innovative method that will allow for novel research and testing of large compound libraries for thyroid cancer drug discovery. However, we also present herein the importance of traditional monolayer culture for deciphering mechanisms of disease progression. Monolayer culture is inexpensive, efficient, and can be highly useful for single-cell mechanistic studies. Our current work shows that the $2 \mathrm{D}$ and $3 \mathrm{D}$ systems represent complementary approaches for uncovering mechanisms of both intrinsic and extrinsic changes in response to drug treatment.

\section{Supplementary materials}

This is linked to the online version of the paper at https://doi.org/10.1530/ ERC-19-0374.

\section{Declaration of interest}

The authors declare that there is no conflict of interest that could be perceived as prejudicing the impartiality of the research reported.

\section{Funding}

This work was funded by the American Cancer Society Institutional Research Grant (\#IRG-58-009-58) and K12 Vanderbilt Clinical Oncology Research Career Development Program (NCI K12CA090625) to V L W; NIH NIGMS R35GM122516 to L M S; NIH NCI R50CA211206 to J A B.

\section{Author contribution statement}

M A L conceived, designed, executed, and interpreted experiments. C J P, S Y B, and K N B designed and executed experiments and provided intellectual input. $\mathrm{K} \mathrm{N} B$ performed the high throughput K1 and TPC1 viability experiments. J A B aided in high-throughput experiments. $L M$

(c) 2020 Society for Endocrinology Published by Bioscientifica Ltd. Printed in Great Britain
S and C J P provided microscopy expertise. V L W provided intellectual guidance and reagents. $\mathrm{V} L \mathrm{~W}$ and $\mathrm{M} \mathrm{A} \mathrm{L} \mathrm{wrote} \mathrm{the} \mathrm{manuscript.}$

\section{Acknowledgements}

The authors thank Dr John Copland for the THJ-11T, THJ-16T, THJ-21T, and THJ-29T thyroid cancer lines and Dr Adel El-Naggar for the TPC1 cell line. The authors would like to acknowledge the Translational Pathology Shared Resource and the High-throughput Screening Shared Resource, supported by NCI/NIH Cancer Center Support Grant 5P30 CA68485-19 and the Vanderbilt Mouse Metabolic Phenotyping Center Grant 2 U24 DK059637-16, and the Shared Instrumental Grant S10 OD023475-01A1 for the Leica Bond RX. The project described was also supported by the National Center for Research Resources, Grant UL1 RR024975-01, and is now at the National Center for Advancing Translational Sciences, Grant 2 UL1 TR000445-06. The content is solely the responsibility of the authors and does not necessarily represent the official views of the $\mathrm{NIH}$.

\section{References}

Anderson RT, Linnehan JE, Tongbram V, Keating K \& Wirth LJ 2013 Clinical, safety, and economic evidence in radioactive iodinerefractory differentiated thyroid cancer: a systematic literature review. Thyroid 23 392-407. (https://doi.org/10.1089/thy.2012.0520)

Brose MS, Nutting CM, Jarzab B, Elisei R, Siena S, Bastholt L, De La Fouchardiere C, Pacini F, Paschke R, Shong YK, et al. 2014 Sorafenib in radioactive iodine-refractory, locally advanced or metastatic differentiated thyroid cancer: a randomised, double-blind, phase 3 trial. Lancet 384 319-328. (https://doi.org/10.1016/S01406736(14)60421-9)

Cabanillas ME, Patel A, Danysh BP, Dadu R, Kopetz S \& Falchook G 2015 BRAF inhibitors: experience in thyroid cancer and general review of toxicity. Hormones and Cancer 6 21-36. (https://doi. org/10.1007/s12672-014-0207-9)

Clevers H 2016 Modeling development and disease with organoids. Cell 165 1586-1597. (https://doi.org/10.1016/j.cell.2016.05.082)

Ely KA, Bischoff LA \& Weiss VL 2018 Wnt signaling in thyroid homeostasis and carcinogenesis. Genes 9 E204. (https://doi. org/10.3390/genes9040204)

Gharib H 2004 Changing trends in thyroid practice: understanding nodular thyroid disease. Endocrine Practice 10 31-39. (https://doi. org/10.4158/EP.10.1.31)

Grebe SK \& Hay ID 1996 Thyroid cancer nodal metastases: biologic significance and therapeutic considerations. Surgical Oncology Clinics of North America 5 43-63. (https://doi.org/10.1016/S10553207(18)30404-6)

Lamouille S, Xu J \& Derynck R 2014 Molecular mechanisms of epithelial-mesenchymal transition. Nature Reviews: Molecular Cell Biology 15 178-196. (https://doi.org/10.1038/nrm3758)

Liang CC, Park AY \& Guan JL 2007 In vitro scratch assay: a convenient and inexpensive method for analysis of cell migration in vitro. Nature Protocols 2 329-333. (https://doi.org/10.1038/nprot.2007.30)

Linder S \& Kopp P 2005 Podosomes at a glance. Journal of Cell Science 118 2079-2082. (https://doi.org/10.1242/jcs.02390)

Lito P, Rosen N \& Solit DB 2013 Tumor adaptation and resistance to RAF inhibitors. Nature Medicine 19 1401-1409. (https://doi. org/10.1038/nm.3392)

Liu R \& Xing M 2016 Tert promoter mutations in thyroid cancer. Endocrine-Related Cancer 23 R143-R155. (https://doi.org/10.1530/ ERC-15-0533)

Mccain J 2013 The MAPK (ERK) pathway: investigational combinations for the treatment of BRAF-mutated metastatic melanoma. Pharmacy and Therapeutics 38 96-108. 
Nusse R \& Clevers H 2017 Wnt/beta-catenin signaling, disease, and emerging therapeutic modalities. Cell 169 985-999. (https://doi. org/10.1016/j.cell.2017.05.016)

Rottner K, Faix J, Bogdan S, Linder S \& Kerkhoff E 2017 Actin assembly mechanisms at a glance. Journal of Cell Science 130 3427-3435. (https://doi.org/10.1242/jcs.206433)

Saito Y, Muramatsu T, Kanai Y, Ojima H, Sukeda A, Hiraoka N, Arai E, Sugiyama Y, Matsuzaki J, Uchida R, et al. 2019 Establishment of patient-derived organoids and drug screening for biliary tract carcinoma. Cell Reports 27 1265.e4-1276.e4. (https://doi. org/10.1016/j.celrep.2019.03.088)

Saito-Diaz K, Chen TW, Wang X, Thorne CA, Wallace HA, Page-Mccaw A \& Lee E 2013 The way Wnt works: components and mechanism. Growth Factors 31 1-31. (https://doi.org/10.3109/08977194.2012.752737)

Sastre-Perona A, Riesco-Eizaguirre G, Zaballos MA \& Santisteban P 2016 Beta-catenin signaling is required for RAS-driven thyroid cancer through PI3K activation. Oncotarget 7 49435-49449. (https://doi. org/10.18632/oncotarget.10356)

Schlumberger MJ 1998 Papillary and follicular thyroid carcinoma. New England Journal of Medicine 338 297-306. (https://doi.org/10.1056/ NEJM199801293380506)
Subbiah V, Kreitman RJ, Wainberg ZA, Cho JY, Schellens JHM, Soria JC, Wen PY, Zielinski C, Cabanillas ME, Urbanowitz G, et al. 2018 Dabrafenib and trametinib treatment in patients With locally advanced or metastatic BRAF V600-mutant anaplastic thyroid cancer. Journal of Clinical Oncology 36 7-13. (https://doi.org/10.1200/ JCO.2017.73.6785)

Takebe T \& Wells JM 2019 Organoids by design. Science 364 956-959. (https://doi.org/10.1126/science.aaw7567)

Vlachogiannis G, Hedayat S, Vatsiou A, Jamin Y, Fernandez-Mateos J, Khan K, Lampis A, Eason K, Huntingford I, Burke R, et al. 2018 Patient-derived organoids model treatment response of metastatic gastrointestinal cancers. Science 359 920-926. (https://doi. org/10.1126/science.aao2774)

Xing M, Liu R, Liu X, Murugan AK, Zhu G, Zeiger MA, Pai S \& Bishop J 2014 BRAF V600E and tert promoter mutations cooperatively identify the most aggressive papillary thyroid cancer with highest recurrence. Journal of Clinical Oncology 32 2718-2726. (https://doi. org/10.1200/JCO.2014.55.5094)

Xu H, Lyu X, Yi M, Zhao W, Song Y \& Wu K 2018 Organoid technology and applications in cancer research. Journal of Hematology and Oncology 11 116. (https://doi.org/10.1186/s13045-018-0662-9)

Received in final form 12 November 2019

Accepted 3 December 2019

Accepted Manuscript published online 5 December 2019 (c) 2020 Society for Endocrinology Published by Bioscientifica Ltd. Printed in Great Britain 